\title{
ON INNER IDEALS AND AD-NILPOTENT ELEMENTS OF LIE ALGEBRAS( ${ }^{(1)}$
}

BY

\author{
GEORGIA BENKART $\left({ }^{2}\right)$
}

\begin{abstract}
ABSTRACr. An inner ideal of a Lie algebra $L$ over a commutative ring $k$ is a $k$-submodule $B$ of $L$ such that $[B[B L]] \subseteq B$. This paper investigates properties of inner ideals and obtains results relating ad-nilpotent elements and inner ideals. For example, let $L$ be a simple Lie algebra in which $D_{y}^{2}=0$ implies $y=0$, where $D_{y}$ denotes the adjoint mapping determined by $y$. If $L$ satisfies the descending chain condition on inner ideals and has proper inner ideals, then $L$ contains a subalgebra $S=\langle e, f, h\rangle$, isomorphic to the split 3-dimensional simple Lie algebra, such that $D_{e}^{3}=D_{f}^{3}=0$. Lie algebras having such 3-dimensional subalgebras decompose into the direct sum of two copies of a Jordan algebra, two copies of a special Jordan module, and a Lie subalgebra of transformations of the Jordan algebra and module. The main feature of this decomposition is the correspondence between the Lie and the Jordan structures. In the special case when $L$ is a finite dimensional, simple Lie algebra over an algebraically closed field of characteristic $p>5$ this decomposition yields: THEOREM. $L$ is classical if and only if there is an $x \neq 0$ in $L$ such that $D_{x}^{p-1}=0$ and if $D_{y}^{2}=0$ implies $y=0$. The proof involves actually constructing a Cartan subalgebra which has 1-dimensional root spaces for nonzero roots and then using the Block axioms.
\end{abstract}

Introduction. One of the most productive concepts in the theory of associative algebras is the notion of an algebra satisfying the descending chain condition on its left or right ideals. Jacobson and McCrimmon have successfully paralleled the Artinian structure theory in their study of Jordan algebras which satisfy the minimum condition on Jordan inner ideals. This paper presents a systematic investigation of inner ideals and ad-nilpotent elements of Lie algebras. It is hoped that inner ideals will play a role analogous to Jordan inner ideals in the development of an Artinian theory for Lie algebras. Many of the definitions used have been coined to imitate the terminology of

Presented to the Society, January 25, 1975; received by the editors August 15, 1975.

AMS (MOS) subject classifications (1970). Primary 17B05, 17B20, 17B65, 17C10, 17C20.

Key words and phrases. Lie algebra, inner ideal, ad-nilpotent element, absolute zero divisor, split 3-dimensional simple Lie algebra, Jordan algebra, Jordan module, Jordan inner ideal, descending chain condition, classical simple Lie algebra.

(') Most of the results of the first two sections of this paper are contained in the author's doctoral dissertation written under the guidance of Professor Nathan Jacobson at Yale University. The author wishes to express her gratitude to Professor Jacobson for his advice and encouragement.

(2) Partially supported by NSF grant GP-33591. 
the Jordan theory. Certain Jordan concepts will come into play in the Lie theory in such a way that there will be meaningful relationships between Lie and Jordan structures bearing the same name.

The preliminary results on inner ideals in the first section yield a classification of minimal inner ideals. The classification gives relations between the ad-nilpotent elements of a Lie algebra and its inner ideals. The focus shifts to Lie algebras which satisfy the minimum condition on inner ideals with the added hypothesis that they contain nonzero ad-nilpotent elements. Suppose such an algebra $L$ has no nonzero ad-nilpotent elements of index less than three. Then:

There is an element $e \neq 0$ in $L$ such that $e \in D_{e}^{2}(L)$ and $D_{e}^{3}=0$, where $D_{e}$ denotes the adjoint mapping determined by $e$.

The second section investigates properties of Lie algebras satisfying the (*)-condition. Such a Lie algebra contains a copy of the split, 3-dimensional simple Lie algebra. The adjoint action of the 3-dimensional subalgebra decomposes the Lie algebra into the direct sum of copies of a Jordan algebra, copies of a Jordan module, and a Lie subalgebra of derivations of the Jordan algebra and module. The main feature of this decomposition is the correspondence between the Lie and Jordan structures. As a consequence of the Jordan structure theory necessary and sufficient conditions are given for the Lie algebra to be the direct sum of a finite number of simple Lie algebras which satisfy the minimum condition on inner ideals.

In the final part the results on Lie algebras which satisfy the (*)-condition are employed to give a simplified criterion using ad-nilpotent elements for distinguishing the nonclassical from the classical Lie algebras over algebraically closed fields of characteristic $p>5$.

1. Inner ideals and ad-nilpotent elements. Throughout this paper the term commutative ring will mean an associative, commutative ring with multiplicative unit. Let $k$ be a commutative ring and assume $L$ is a Lie algebra over $k$. A $k$-submodule $B$ of $L$ is an inner ideal of $L$ if $[B[B L]] \subseteq B$. Every ideal is an inner ideal, but simple Lie algebras can have nontrivial inner ideals. For example, consider the Lie algebra $s l(2, F)$ of $2 \times 2$ matrices of trace zero over a field $F$ of characteristic not 2 . Then

$$
e=\left(\begin{array}{ll}
0 & 1 \\
0 & 0
\end{array}\right), f=\left(\begin{array}{ll}
0 & 0 \\
1 & 0
\end{array}\right) \text { and } h=\left(\begin{array}{cc}
1 & 0 \\
0 & -1
\end{array}\right)
$$

form a basis, and the subspaces $F e$ and $F f$ are inner ideals.

Let $D_{a}$ denote the adjoint mapping determined by $a$. For additive subgroups $A, B, C$ of $L$ let $A B=[A, B]$ and $A B C=[A, B C]$. Define $A_{1} \cdots A_{r}$ inductively by $A_{1} \cdots A_{r}=\left[A_{1}, A_{2} \cdots A_{r}\right]$. In this notation the lower central 
series of $A$ is given by $A^{1}=A, A^{2}=A A$, and $A^{r}=A A^{r-1}$. In the new notation a $k$-submodule $B$ of $L$ is an inner ideal of $L$ provided $B B L \subseteq B$.

LEMMA 1.1. Assume $B$ is an inner ideal of $L$.

(1) $B B L$ is an inner ideal and subalgebra of $L$ as well as an ideal of $B$.

(2) $L B^{n} \subseteq B^{n-1}$ for $n \geqslant 2$.

(3) If $B^{2}=B$, then $B$ is an ideal of $L$.

(4) For every $k$-submodule $V$ of $B$ with $V V B \subseteq V, V^{3}$ is an inner ideal of $L$.

(5) For every additive subgroup $V$ of $B, V^{2} V^{2} L \subseteq V V B$.

Proof. (1) Since $B B L \subseteq B$,

$$
[B B L[B B L, L]] \subseteq[B[B L]]=B B L,
$$

and

$$
[B B L, B B L] \subseteq[B, B B L]=[B[B, B L]] \subseteq B B L
$$

The proof of (2) follows by induction on $n$, and these standard results for additive subgroups $U, V, W, X$ of $L$ :

$$
U V W \subseteq V W U+W U V \text {, and }[U V, W X] \subseteq U V W X+V U W X
$$

Part (3) is a consequence of (2) with $n=2$. To prove (4) it suffices to look at the mapping $D_{\left[u^{\prime \prime}\left[u^{\prime}, u\right]\right]} D_{\left[v^{\prime \prime}\left[v^{\prime}, v\right]\right]}$ applied to $L$ for $u$ 's, $v^{\prime}$ ' in $V$. But this mapping is the sum of terms of the form $D_{a} D_{b} D_{c} D_{d} D_{e} D_{f}$ where $a, \ldots, f \in V$. Now $D_{e} D_{f}(L) \subseteq B, D_{c} D_{d}(B) \subseteq V$, and $D_{a} D_{b}(V) \subseteq V^{3}$. A similar argument gives (5).

This lemma shows that for each inner ideal $B$ of $L$ there is a descending chain of inner ideals given by $B_{0}=B, B_{1}=B B L, B_{2}=B_{1} B_{1} L, \ldots$ Moreover (4) applied to $V=B$ shows $B^{3}$ is an inner ideal whenever $B$ is. Still another way of manufacturing inner ideals is to begin with an arbitrary additive subgroup $A$ of $L$ and to define $T(A)$ to be $\{t \in L \|[A[L]] \subseteq A\}$.

LEMMA 1.2. $T(A)$ is an inner ideal and subalgebra of $L$ which contains $A$ if $A$ is an inner ideal of $L$.

Proof. That $T(A)$ contains $A$ whenever $A$ is an inner ideal follows immediately from the definition of $T(A)$. To show $T(A)$ is itself an inner ideal let $s, t \in T(A), x, y \in L, a \in A$. Then

$$
\begin{aligned}
{[a[[t[s, x]] y]] } & =[a[t[[s, x] y]]]+[a[[t, y][s, x]]] \\
& \equiv[a[[t, y][s, x]]] \bmod A \\
& \equiv[[a[t, y]][s, x]]+[[t, y][a[s, x]]] \bmod A \\
& \equiv 0 \bmod A .
\end{aligned}
$$


The arguments that $T(A)$ is a subalgebra and a $k$-submodule are of a similar nature.

The inner ideals $T(A)$ defined in this manner often enable us to reduce our considerations to inner ideals which are also subalgebras for which more can be said:

LEMMA 1.3. Let $T$ be an inner ideal and subalgebra of $L$. Then for all $n \geqslant 1$, $T^{n}$ is an inner ideal of $L$ and $\cap_{n=1}^{\infty} T^{n}$ is an ideal of $L$.

Proof. The lemma is a consequence of Lemma 1.1, part (2).

An inner ideal $B \neq(0)$ is said to be minimal if there exists no inner ideal $C$ of $L$ such that $B \supset C \supset(0)$. Trivially, $F e$ and $F f$ are minimal inner ideals of $s l(2, F)$, though it is not true in general that every minimal inner ideal is 1-dimensional. An element $z$ is an absolute zero divisor of $L$ if $D_{z}^{2}=0$. An absolute divisor generates an inner ideal which will be minimal if $k$ is a field. Absolute zero divisors cannot exist in finite dimensional semisimple Lie algebras over fields of characteristic 0 because every ad-nilpotent element can be imbedded in a subalgebra isomorphic to $s l(2, k)$ as seen in Jacobson [10, p. 100]. One can verify readily that $s l(2, k)$ has no absolute zero divisors. However, there do exist finite dimensional simple Lie algebras over algebraically closed fields of prime characteristic such as the Witt algebra having absolute zero divisors. The role of absolute zero divisors has been explored previously in the work of Kostrykin [14], [15]. They appear to distinguish the classical Lie algebras from the nonclassical over algebraically closed fields of characteristic $p>5$. The final section of this paper will demonstrate further results along these lines.

THEOREM 1.4. Let $B$ be a minimal inner ideal of a Lie algebra $L$ over a commutative ring $k$. Then either:

(1) $B=k b$ where $b$ is an absolute zero divisor of $L$, or

(2) $B=B B L$ and $B^{2}=(0)$, or

(3) $B$ is a simple ideal of $L$ and for every proper inner ideal $V$ of $B, V^{2}=(0)$.

Proof. If $B$ contains a nonzero absolute zero divisor $B$, the minimality of $B$ gives $B=k b$. So we can assume $B$ has no absolute zero divisors different from zero. Since $B B L$ is an inner ideal of $L$ contained in $B, B B L=B$ or $B B L=(0)$. But the last possibility can be excluded because $B$ has no nonzero absolute zero divisors. So $B=B B L$ and by Lemma $1.1 B$ is a subalgebra of $L$. By the remarks following Lemma 1.1, there are two possibilities: $B^{3}=0$ or $B^{3}=B$. In the first case Lemma 1.1(5) applied to $B$ gives $B^{2} B^{2} L \subseteq B^{3}=(0)$. That is to say, every element of $B^{2}$ is an absolute zero divisor of $L$. Since $B$ is a subalgebra $B^{2} \subseteq B$, so $B^{2}=(0)$ must necessarily hold. We may suppose then that $B B L=B$ and also $B^{3}=B$. Because $B$ is a subalgebra $B \supseteq B^{2} \supseteq$ 
$B^{3}=B$ and $B^{2}=B$. According to Lemma 1.1(2), $B$ is an ideal of $L$. Any ideal of $B$ is an inner ideal of $L$, so $B$ is simple. For any inner ideal $V$ of $B$ with $V \neq(0)$ or $B$ we have

$$
V^{2} V^{2} L \subseteq V^{2} V B \subseteq V V V B \subseteq V^{2}
$$

showing $V^{2}$ is an inner ideal of $L$ contained in $B$. If $V^{2}=B$, then $V^{2} \supseteq V$ and $V \supseteq V^{3} \supseteq V^{2}=B$, contrary to assumption. So it must be for every inner ideal $V$ of $B$ that $V^{2}=(0)$.

For any inner ideal $B$ of $L$ such that $B^{2}=(0)$, if $b \in B$ then $D_{b}^{3}=0$. If $V$ is an inner ideal of an ideal $B$ with $V^{2}=(0)$, then $D_{v}^{4}=0$ for $v \in V$. Thus Theorem 1.4 shows that the existence of minimal inner ideals implies the existence of ad-nilpotent elements (of index $<4$ ) except when every minimal inner ideal is a simple ideal having no proper inner ideals. In particular, if a simple Lie algebra has proper minimal inner ideals, it has ad-nilpotent elements. On the other hand as we see next, the existence of ad-nilpotent elements generally implies the existence of nontrivial inner ideals.

Let $L$ be a Lie algebra over a commutative ring $k$ and let $n$ be an integer $>2$. We say $L$ is $n$-torsion free if, for $x \in L, n x=0$ implies $x=0$. Kostrykin [12] has proved the following fundamental result concerning adnilpotent elements in $L$.

Proposition 1.5. Suppose $D_{a}^{m}=0$ for $a \neq 0$ and $m>3$. If $L$ is $n$-torsion free for all $n \leqslant m$, then

$$
\left(D_{D_{a}^{m-1}(c)}\right)^{m-1}=0 \text { for all } c \in L .
$$

COROLLARY 1.6. If $a$ and $L$ are as in the preceding proposition, there is a $b \neq 0$ in $L$ with $D_{b}^{3}=0$.

LEMMA 1.7. Let $L$ be a 3-torsion free Lie algebra and suppose $a \in L$ with $D_{a}^{3}=0$. Let $A=D_{a}$ and for $x \in L$ let $X=D_{x}$. Then:

$$
\begin{aligned}
A^{2} X A & =A X A^{2}, \\
A^{2} X A^{2} & =0, \\
D_{A^{2}(x)}^{2} & =A^{2} X^{2} A^{2}, \\
D_{A^{2}(x)}^{3} & =0 .
\end{aligned}
$$

Proof. Because $A^{3}(x)=0$ for all $x \in L, 0=D_{A^{3}(x)}=[A[A[A X]]]$. From this we see $-3 A^{2} X A+3 A X A^{2}=0$, and since $L$ is 3-torsion free (i) follows. Multiplying (i) on the right by $A$ gives (ii). Now

$$
D_{A^{2}(x)}^{2}=\left(A^{2} X-2 A X A+X A^{2}\right)^{2} .
$$

so (i) and (ii) can be used to prove (iii). Finally the last statement holds because 


$$
D_{A^{2}(x)}^{3}=D_{A^{2}(x)} D_{A^{2}(x)}^{2}=\left(A^{2} X-2 A X A+X A^{2}\right) A^{2} X^{2} A^{2}=0 .
$$

LEMMA 1.8. Let $L$ be 3-torsion free and $a \in L$ be such that $D_{a}^{3}=0$. Then $D_{a}^{2}(L)$ is an inner ideal of $L$, and $D_{a}^{2}(L)$ is abelian.

Proof. It suffices to show $D_{x} D_{y}(L) \subseteq D_{a}^{2}(L)$ for $x=D_{a}^{2}(b)$ and $y=$ $D_{a}^{2}(c)$. Let $A=D_{a}, B=D_{b}, C=D_{c}$. Then

$$
D_{x} D_{y}=\left(A^{2} B-2 A B A+B A^{2}\right)\left(A^{2} C-2 A C A+C A^{2}\right) \text {. }
$$

Using (ii) above we eliminate the terms involving $A^{2} B A^{2}$ and $A^{2} C A^{2}$. Thus,

$$
\begin{aligned}
D_{x} D_{y}= & -2\left(A^{2} B A\right) C A+2\left(A B A^{2}\right) C A+A^{2} B C A^{2} \\
& +2 A B\left(A^{2} C A\right)-A B\left(A C A^{2}\right) .
\end{aligned}
$$

Equation (i) applied to this gives

$$
D_{x} D_{y}=A^{2} B C A^{2} \text {. }
$$

Consequently, $D_{x} D_{y}(L) \subseteq D_{a}^{2}(L)$, and $D_{a}^{2}(L)$ is an inner ideal of $L$ which is abelian since

$$
D_{x}(y)=\left(A^{2} B-2 A B A+B A^{2}\right)\left(A^{2}(c)\right)=0 .
$$

This lemma shows that the existence of an ad-nilpotent element $a \neq 0$ of index $<3$ implies the existence of a proper inner ideal unless $D_{a}^{2}(L)=0$ or $D_{a}^{2}(L)=L$. The nilpotency of $D_{a}$ rules out the second case, and in the first case $k a$ is a proper inner ideal except when $L=k a$. To summarize:

LEMMA 1.10. Let $L$ be a 3-torsion free Lie algebra. If $D_{a}^{3}=0$ for some $a \neq 0$ in $L$, then $L$ has a proper inner ideal unless $L=k z$.

CoROLlaRY 1.11. Let $L$ be a Lie algebra and $0 \neq a \in L$ with $D_{a}^{m}=0$. If $L$ is $n$-torsion free for $n \leqslant m$, then $L$ has a proper inner ideal unless $L=k z$.

These considerations yield the following strengthening of the minimal inner ideal theorem:

THEOREM 1.12. Let $B$ be a minimal inner ideal of a Lie algebra $L$ which is 2 and 3-torsion free. Then either:

(1) $B=k b$ where $b$ is an absolute zero divisor of $L$, or

(2) $B=D_{b}^{2}(L)$ for all $b \neq 0$ in $B$, and $B^{2}=(0)$, or

(3)' $B$ is an ideal which is simple as a Lie algebra and which contains no proper inner ideals.

Proof. Only (3)' needs some explanation. Recall (3) in the original theorem is the case when $B$ is a simple ideal of $L$ such that $V^{2}=(0)$ for every inner ideal $V \neq B$ of $B$. For every $v$ in such a $V, D_{v}^{4}=0$, so that by Kostrykin's result, for any $x \in L$ if $u=D_{v}^{3}(x)$, then $D_{u}^{3}=0$. Since $u \in B, D_{u}^{2}(L)$ is an inner ideal of $L$ contained in $B$, and hence must be (0) or $B$. Using $D_{u}^{3}=0$ 
and the fact that $B$ is an ideal of $L$, we note that $D_{u}^{2}(L)=B$ implies $B=D_{u}^{2}(L)=(0)$, a contradiction. The case $D_{u}^{2}(L)=0$ gives $u=0$, and thus $D_{0}^{3}=0$. By the same reasoning applied to $v$ we obtain $V=(0)$.

We say a Lie algebra is Artinian if every descending chain of inner ideals terminates. The term Artinian Lie algebra is well chosen for if $R$ is a simple Artinian ring of characteristic not 2 or 3 with center $Z$, then $[R R] /[R R] \cap Z$ is a simple Artinian Lie algebra. If $R$ has an involution * and if $K$ is the set of skew elements relative to *, then $[K K] /[K K] \cap Z$ is a simple Artinian Lie algebra provided the dimension of $R$ over $Z$ is greater than 16. (Proofs of these statements appear in [2].) We shall call a Lie algebra $L$ nondegenerate if it contains no absolute zero divisors different from zero. One justification for calling these algebras nondegenerate is the fact that if $L$ is a finite dimensional Lie algebra with nonzero absolute zero divisors, then the Killing form is degenerate, as seen in Kostrykin [13]. The next result will not be used in the remainder of the paper; however, it illustrates the way Lemma 1.2 can be used to study arbitrary inner ideals.

Lemma 1.13. Let $L$ be a nondegenerate, simple, Artinian Lie algebra. For every inner ideal $V \neq L$ of $L, V^{2}=(0)$.

Proof. Assume initially that $V$ is also a subalgebra. By Lemma 1.3, $\cap_{n=1}^{\infty} V^{n}$ is an ideal of $L$ which must then be (0). The subalgebras $V=V^{1} \supseteq$ $V^{2} \supseteq \ldots$ form a descending chain of inner ideals. Therefore there is an integer $m$ such that $V^{m}=\bigcap_{n=1}^{\infty} V^{n}=(0)$. If $m=2$, then $V^{2}=(0)$, the desired conclusion. If $m>2$, then

$$
V^{m-1} V^{m-1} L \subseteq V^{m-1} V^{m-2} \subseteq V^{m}=(0) .
$$

Every element of $V^{m-1}$ is an absolute zero divisor of $L$. Thus $V^{m-1}=(0)$, and an inductive argument shows $V^{2}=(0)$. Now if $V \neq L$ is an arbitrary inner ideal, define $T(V)$ as above. Since $T(V)$ is an inner ideal and subalgebra of $L$ containing $V, T(V)=L$ or $T(V)^{2}=(0)$. In the last case $V^{2}=(0)$. However, if $T(V)=L$, then $V L L \subseteq V$. Because $L$ is simple $L=L^{2}$ and so $V L \subseteq V$. This says $V$ is an ideal of $L$ which is impossible. So only the case $V^{2}=(0)$ can occur.

The lemma can be applied to finite dimensional simple Lie algebras over fields of characteristic 0 or to classical Lie algebras over fields of characteristic $p>3$ to show inner ideals of these algebras are necessarily abelian.

We now investigate nondegenerate, Artinian Lie algebras having ad-nilpotent elements. Such Lie algebras will be shown to contain a copy of $s l(2, k)$. Results derived from studying the adjoint representation of this subalgebra on the Lie algebra will be the main topic of the next section.

Lemma 1.14. Let $L$ be a nondegenerate, Artinian Lie algebra. Assume there is 
an $a \neq 0$ in $L$ with $D_{a}^{m}=0$ for some $m \geqslant 3$, and that for $n<m, L$ is $n$-torsion free. Then there is an $e \neq 0$ in $L$ with $D_{e}^{3}=0$ and $e \in D_{e}^{2}(L)$. If $\frac{1}{2}, \frac{1}{3} \in k$, then $L$ contains a copy of $s l(2, k)$.

Proof. By Kostrykin's result we can suppose that there is an element $0 \neq b$ in $L$ with $D_{b}^{3}=0$. For such an element $D_{b}^{2}(L)$ is an inner ideal of $L$ which is nonzero because $L$ is nondegenerate. The collection of all such inner ideals is nonempty, and so must possess a minimal element, say $D_{d}^{2}(L)$. Let $e \neq 0$ be in $D_{d}^{2}(L)$ and observe that from (iv) of Lemma $1.7, D_{e}^{3}=0$. From (iii) of that lemma $0 \neq D_{e}^{2}(L) \subseteq D_{d}^{2}(L)$. Minimality then implies $e \in D_{d}^{2}(L)=D_{e}^{2}(L)$. Assume now $h \in D_{e}(L)$ is chosen so that $[h e]=2 e$. If $\frac{1}{2}, \frac{1}{3} \in k$, then Seligman [14, p. 122] has shown there is an $f \in L$ with $[h f]=-2 f$ and $[e f]=h$. The subalgebra generated by $e, f, h$ is isomorphic to $s l(2, k)$.

We will say a Lie algebra is a *-Lie algebra if it satisfies the following condition:

$$
\text { There is an } e \neq 0 \text { in } L \text { with } D_{e}^{3}=0 \text { and } e \in D_{e}^{2}(L) \text {. }
$$

The preceding lemma shows every nondegenerate, Artinian Lie algebra which has an ad-nilpotent element and which is suitably torsion-free is a ${ }^{*}$-Lie algebra. In particular, every finite dimensional semisimple Lie algebra over a field of characteristic 0 which has a nonzero ad-nilpotent element is a ${ }^{*}$-Lie algebra.

2. *-Lie algebras. Throughout this section $L$ will be a *-Lie algebra over a commutative ring $k$ such that $\frac{1}{2}, \frac{1}{3} \in k$.

LEMMa 2.1. There exist $k$-submodules $V_{i}$ such that

(1) $L=V_{2} \oplus V_{1} \oplus V_{0} \oplus V_{-1} \oplus V_{-2}$,

(2) $V_{0}$ decomposes as the sum of submodules $V_{0}=Z \oplus T$,

(3) $V_{2}, V_{-2}, T$ are isomorphic as $k$-submodules of $L$ as are $V_{1}, V_{-1}$, and $Z$ is a subalgebra of $L$.

Proof. Suppose $e \neq 0$ in $L$ is such that $D_{e}^{3}=0$ and $e \in D_{e}^{2}(L)$. Let $h, f$ be chosen as in $\S 1$. Then $H=D_{h}$ is algebraic over $k$ with minimum polynomial a factor of $p(\lambda)=(\lambda-2)(\lambda-1) \lambda(\lambda+1)(\lambda+2)$ (see for example Jacobson [8] or [9]). Let $p_{i}(\lambda)=p(\lambda) /(\lambda+i)$ and observe:

$$
1=\frac{1}{24} p_{2}(\lambda)-\frac{1}{6} p_{1}(\lambda)+\frac{1}{4} p_{0}(\lambda)-\frac{1}{6} p_{-1}(\lambda)+\frac{1}{24} p_{-2}(\lambda) .
$$

As a consequence we can decompose $L$ as a $k$-module into eigenspaces relative to $H$. Thus $L=V_{2} \oplus V_{1} \oplus V_{0} \oplus V_{-1} \oplus V_{-2}$ where $H v_{i}=i v_{i}$ for $v_{i} \in V_{i}$. Because $H$ is a derivation, $\left[V_{i}, V_{j}\right] \subseteq V_{i+j}$ where $V_{m}=(0)$ if $m>2$ or $m<-2$. Let $E=D_{e}$ and $F=D_{f}$. The mapping $\frac{1}{2} E^{2}: V_{-2} \rightarrow V_{2}$ is a $k$-isomorphism with inverse $\frac{1}{2} F^{2}$. Also $V_{-1}$ and $V_{1}$ are $k$-isomorphic under 
the map $E$ which has inverse $F$. Define a map $\sigma$ on $V_{0}$ by $\sigma(v)=v-E F(v)$. One verifies readily that $\sigma^{2}(v)=v$ for all $v \in V_{0}$. Let $Z=\left\{v \in V_{0} \mid \sigma(v)=\right.$ $v\}$ and $T=\left\{v \in V_{0} \mid \sigma(v)=-v\right\}$. Then $V_{0}=Z \oplus T$ and the map $E$ is a $k$-isomorphism sending $T$ to $V_{2}$. If $\sigma(v)=v$, then $E F(v)=0$. This implies $0=F E F(v)=-H F(v)=F(v)$. Similarly $E(v)=0$, and from this it follows that $Z$ is just the centralizer of the subalgebra generated by $e, f, h$; hence itself is a subalgebra.

Let us note that for $v \in V_{0}$, if $E(v)=0$, then $E F(v)=F E(v)+H(v)=$ 0 and $v \in Z$. Similarly $v \in V_{0}$ and $F(v)=0$ implies $v \in Z$.

Adopt the notation $A=V_{2}, \bar{A}=V_{-2}, \bar{a}=-\frac{1}{2} F^{2}(a) \in \bar{A}$ and $a^{*}=$ $-\frac{1}{2} F(a) \in T$ for $a \in A$. For $a, b \in A$ define a product on $A$ by $a \cdot b$ $=\frac{1}{2}[a[f b]]$, and for $z \in Z$ let $z(a)=[z, a]$.

LEMMA 2.2. $A$ is a Jordan algebra relative to this product and $Z$ acts as derivations on $A$.

Proof. To begin let us observe that the product in $A$ is commutative since $[a, b]=0$. Because $\frac{1}{2}[a[f e]]=\frac{1}{2}[h a]=a$, the element $e$ acts as the identity in $A$. What remains to be shown is the Jordan identity and the last assertion. Since $Z$ centralizes $f$, it is clear that $\left[z, a^{*}\right]=z(a)^{*},[z, a]=\overline{z(a)}$, and $z(a \cdot b)=z(a) \cdot b+a \cdot z(b)$. As a further consequence of the definition we have $\left[a^{*}, b\right]=a \cdot b=\left[b^{*}, a\right]$. The equation

$$
[f, a \cdot b]=\frac{1}{2}[f[a[f, b]]]=\frac{1}{2}[[f a][f b]]+\left[a, \frac{1}{2}[f[f b]]\right],
$$

when rewritten in the above notation, gives an expression for $[a, \bar{b}]$, namely:

$$
[a, \bar{b}]=2(a \cdot b)^{*}+2\left[a^{*}, b^{*}\right] \text {. }
$$

Now $\left[a^{*}, b^{*}\right] \in V_{0}$ and $\left[\left[a^{*}, b^{*}\right] e\right]=0$. So by the above remarks $\left[a^{*}\right.$, $\left.b^{*}\right] \in Z$. Applying $D_{f}$ to both sides of $(2.3)$ shows $\left[a^{*}, \bar{b}\right]=-\overline{a \cdot b}$. Using these results we calculate the Jacobi identity for $a, \bar{b}, c^{*}$ and obtain

$$
\left[(a \cdot b)^{*}, c^{*}\right]+\left[(b \cdot c)^{*}, a^{*}\right]+\left[(c \cdot a)^{*}, b^{*}\right]=0 \text {. }
$$

The element $a^{*}$ acts as left multiplication by $a$ on $A$. Therefore if $a=b=c$ in (2.4), $\left[\left(a^{2}\right)^{*}, a^{*}\right]=0$ and this shows $A$ is a Jordan algebra.

As yet we have ignored the spaces $V_{1}, V_{-1}$ which may in fact be zero. This particular case has been studied previously in the work of Tits [19] and Hirzebruch [6] for fields, and for commutative rings in an unpublished work of Jacobson. In the situation in which $V_{1}$ occurs, let $a \cdot v=[a[f v]]$ and $z(v)=[z, v]$ for $a \in A, v \in V_{1}, z \in Z$. In light of the isomorphism between $V_{1}$ and $V_{-1}$ let us adopt the notation $\bar{v}=[f v], V=V_{1}$ and $\bar{V}=V_{-1}$.

LEMMA 2.5. $V$ is a special unital $A$-module with skew bilinear mapping

$$
V \times V \stackrel{\leftrightarrow,\rangle}{\rightarrow} A
$$


and trilinear product

$$
V \times V \times V^{\langle,,\rangle} \rightarrow V
$$

so that $(A, V)$ is a J-ternary algebra in the sense of Allison [1].

Proof. From the definition of $a \cdot v$ it is clear that $e \cdot v=v$ and $[a$, $v]=a \cdot v$. It is also immediate that $\left[a^{*}, v\right]=\frac{1}{2} a \cdot v$, and applying $D_{f}$ to both sides of this equation shows $[\bar{a}, v]=\bar{a} \cdot v$. The effect of $a^{*}$ on $\bar{v}$ is given by

$$
\left[a^{*}, \bar{v}\right]=\frac{1}{2}[[a, f] \bar{v}]=\frac{1}{2}[[a, \bar{v}] f]=\frac{1}{2}[a \cdot v, f]=-\frac{1}{2} \overline{a \cdot v} \text {. }
$$

We are now in a position to calculate the action of the product $a \cdot b$ on $V$. For

$$
\begin{aligned}
(a \cdot b) \cdot v & =\left[\left[a^{*}, b\right] \bar{v}\right]=\left[\left[a^{*}, \bar{v}\right] b\right]+\left[a^{*}[b, \bar{v}]\right] \\
& =\frac{1}{2} b \cdot(a \cdot v)+\frac{1}{2} a \cdot(b \cdot v) .
\end{aligned}
$$

That is, $V$ is a special unital $A$-module. The commutator in $L$ defines a skew bilinear map $\langle$,$\rangle of V \times V$ into $A$, and the Jacobi identity applied to $a^{*}, u, v$ yields

$$
a \cdot\langle u, v\rangle=\frac{1}{2}\langle a \cdot u, v\rangle+\frac{1}{2}\langle u, a \cdot v\rangle .
$$

We define $\langle u, v, w\rangle$ to be $[u[v, w]] \in V$, and compute $\left[a^{*},\langle u, v, w\rangle\right]$ to show

$$
a \cdot\langle u, v, w\rangle=\langle a \cdot u, v, w\rangle+\langle u, a \cdot v, w\rangle-\langle u, v, a \cdot w\rangle .
$$

Now Axiom (Tl) in [1] is just the specialization of the Jacobi identity with $u, v, \bar{w}$. From the Jacobi identity with $f, v, w$ we have $-2\langle v, w\rangle^{*}=[v$, $\bar{w}]-[w, v]$. Taking the product of both sides with $u$ shows (T2) holds. Condition (T3) is a consequence of calculating $[[u[v, w]] x]$. Finally by applying (T2) we see $[e[\bar{w}[x, y]]]=\langle w, y, x\rangle$. Thus, $[\bar{w}[x, y]]=\langle\overline{w, y, x}\rangle$. Using this fact (T4) results from computing $[[u[v, w]],[x y]]$. (Compare Theorem 1 in Allison [1] and results in Hein [5].)

Let us observe that $Z$ acts as derivations on $V$ in the sense that $z(a \cdot v)=$ $z(a) \cdot v+a \cdot z(v)$. The element $D(u, v)=\frac{1}{2}\{[u v]+[v u]\}$ belongs to $V_{0}$ and commutes with $e$ so it is in $Z$. Therefore the product [uv] is given by $[u v]=D(u, v)-\langle u, v\rangle^{*}$. The elements $D(u, v)$ will be useful later in describing ideals in a ${ }^{*}$-Lie algebra.

To discuss the relationship between the Lie and Jordan structures in *-Lie algebras some Jordan concepts are needed. Assume $A$ is a Jordan algebra over a commutative ring $k$. A $k$-submodule $B$ of $A$ is a Jordan inner ideal of $A$ if $U_{b_{1}, b_{2}}(a) \in B$ for every $b_{1}, b_{2} \in B, a \in A$, where

$$
U_{b_{1}, b_{2}}(a)=\left(b_{2} \cdot a\right) \cdot b_{1}+\left(a \cdot b_{1}\right) b_{2}-a \cdot\left(b_{1} \cdot b_{2}\right) \text {. }
$$

If $a^{*}$ denotes left multiplication by $a$, then 


$$
U_{b_{1}, b_{2}}(a)=\left[b_{1}^{*}, a^{*}\right]\left(b_{2}\right)+\left(b_{1} \cdot a\right)^{*}\left(b_{2}\right) .
$$

An element $b$ of $A$ is an absolute zero divisor if $U_{b, b}=0$. Clearly an absolute zero divisor generates a Jordan inner ideal which is minimal if $k$ is a field. The results for minimal Jordan inner ideals (Jacobson [11, p. 154]) bear a striking resemblance to the theorem on minimal Lie inner ideals in the first section.

Suppose now that $L=A \oplus V \oplus Z \oplus A^{*} \oplus \bar{V} \oplus \bar{A}$ is a *-Lie algebra, and let $B$ be an inner ideal of $L$ contained in $A$. Such inner ideals exist since $A$ itself is an inner ideal of $L$. For $b_{1}, b_{2} \in B, a \in A,\left[\left[b_{1}, a\right] b_{2}\right] \in B$, but

$$
\left[\left[b_{1}, \bar{a}\right] b_{2}\right]=2\left[b_{1}^{*}, a^{*}\right]\left(b_{2}\right)-2\left(b_{1} \cdot a\right)^{*}\left(b_{2}\right)=2 U_{b_{1}, b_{2}}(a)
$$

according to (2.3). Thus $B$ is a Jordan inner ideal of $A$, and conversely any Jordan inner ideal of $A$ is an inner ideal of $L$.

Lemma 2.6. Let $L=A \oplus V \oplus A^{*} \oplus Z \oplus \bar{V} \oplus \bar{A}$ be $a^{*}$-Lie algebra.

(1) If $L$ is Artinian, then $A$ satisfies the minimum condition on Jordan inner ideals.

(2) If $L$ is nondegenerate, then $A$ has no absolute zero divisors.

(3) If $L$ is nondegenerate and Artinian, then $A$ is the direct sum of a finite number of simple ideals which satisfy the minimum condition on Jordan inner ideals, and for each $a \in A, a \in D_{a}^{2}(L)$ and $D_{a}^{3}=0$.

Proof. Only (3) needs discussion. One knows from McCrimmon [16] that under the assumption of the minimum condition for Jordan inner ideals the condition "no absolute zero divisors" is equivalent to the Jordan algebra being regular. That is, $A$ is regular if for each $a \in A$ there is a $b$ in $A$ with $U_{a, a}(b)=a$. Regularity and the minimum condition imply the Jordan algebra is the direct sum of simple ideals which then must also satisfy the minimum condition. The last assertions follow from the regularity of $A$ and from the fact that $\left[V_{i}, V_{j}\right] \subseteq V_{i+j}$. A similar result holds for each $\bar{a} \in \bar{A}$.

Lemma 2.7. Let $L=A \oplus V \oplus A^{*} \oplus Z \oplus \bar{V} \oplus \bar{A}$ be a nondegenerate, Artinian *-Lie algebra such that the $k$-submodule generated by $h=2 e^{*}$ is a maximal split torus of $L$, then $A$ is a Jordan division algebra.

Proof. For $0 \neq a \in A$ since $D_{a}^{3}=0$ and $a \in D_{a}^{2}(L)$, then there is a $b \in A$ with $[a, \bar{b}]=h^{\prime},\left[h^{\prime} \bar{b}\right]=-2 \bar{b}$, and $\left[h^{\prime} a\right]=2 a$. This implies $D_{h^{\prime}}$ is a semisimple transformation with eigenvalues among $\pm 2, \pm 1,0$. Because $h^{\prime}$ centralizes $h$, the maximality of the torus forces $h^{\prime}$ to be a nonzero multiple of $h$. That is,

$$
h^{\prime}=[a, \bar{b}]=2\left[a^{*}, b^{*}\right]+2(a \cdot b)^{*}=2 \alpha e^{*} .
$$

Therefore $\left[a^{*}, b^{*}\right]=0$ and $a \cdot b=\alpha e$. But $2 a=[[a \bar{b}] a]=2 \alpha a$ so that $\alpha=1$ and $a \cdot b=e$. Then 


$$
\begin{aligned}
2 a & =[[a \bar{b}] a]=2\left[a^{*}, b^{*}\right](a)+2(a \cdot b)^{*}(a) \\
& =2\left(a-b \cdot a^{2}+a\right),
\end{aligned}
$$

giving $a=b \cdot a^{2}$. These two conditions say $A$ is a Jordan division algebra.

In general it is not true that for a nondegenerate, Artinian *-Lie algebra that the Jordan algebra is necessarily a division algebra. However, as we see in the next lemma, there is some decomposition of the Lie algebra so that the Jordan algebra is a division algebra.

Lemma 2.8. Let $L$ be a nondegenerate, Artinian *-Lie algebra. Then $L=A^{\prime}$ $\oplus V^{\prime} \oplus A^{\prime *} \oplus Z^{\prime} \oplus \overline{V^{\prime}} \oplus \overline{A^{\prime}}$ where $A^{\prime}$ is a Jordan division algebra.

Proof. Consider the collection of all inner ideals of the form $D_{c}^{2}(L)$ where $D_{c}^{3}=0$. It is nonempty and so possesses a minimal element, say $A^{\prime}$. By the same argument used in the proof of Lemma 1.14, there is an $e^{\prime} \in A^{\prime}$ with $e^{\prime} \in D_{e^{\prime}}^{2}(L)=A^{\prime}$ and $D_{e^{\prime}}^{3}=0$. Then $L=A^{\prime} \oplus V^{\prime} \oplus A^{\prime *} \oplus Z^{\prime} \oplus \overline{V^{\prime}} \oplus \overline{A^{\prime}}$ and for each $a \in A^{\prime}, D_{a}^{2}(L)=A^{\prime}$. Because $A^{\prime}$ is a minimal inner ideal of $L$, $A^{\prime}$ is a Jordan algebra without proper Jordan inner ideals. One can see from the Jordan minimal inner ideal theorem that $A^{\prime}$ must be a division algebra.

The Lie and Jordan correspondence can be further evidenced in the study of ideals of a ${ }^{*}$-Lie algebra.

LEMMA 2.9. Let $L$ be an arbitrary *-Lie algebra and $J$ be an ideal of $L$. Then $J=I \oplus W \oplus Y \oplus I^{*} \oplus \bar{W} \oplus \bar{I}$ where:

(i) $I$ is an ideal of $A$ such that $Z(I) \subseteq I$;

(ii) $W$ is an $A$-submodule of $V$ with $Z(W) \subseteq W$ and $I V \subseteq W \subseteq\{v \in V \mid$ $\langle v, V\rangle \subseteq I\}$;

(iii) $Y$ is an ideal of $Z$ and

$$
D(V, W)+\left[A^{*}, I^{*}\right] \subseteq Y \subseteq\{z \in Z \mid z(A) \subseteq I, z(V) \subseteq W\} .
$$
$L$.

Any $k$-submodule formed by $I, W, Y$ satisfying these properties is an ideal of

Proof. Since $J$ is stable under the action of the subalgebra generated by $e$, $f=\bar{e}, h=2 e^{*}, J$ decomposes into submodules as claimed. The remaining assertions can be verified using results derived in the proof of Lemmas 2.2 and 2.5. Let us note that in (ii) we may choose $W=I V$ or $W=\{v \in V \mid\langle v$, $V\rangle \subseteq I\}$. Likewise in (iii) $Y$ may be taken to be either extreme. In particular, if $R=\{v \in V \mid\langle v, V\rangle=0\}$, then $R+Y+\bar{R}$ is an ideal where $Y$ is any ideal of $Z$ which satisfies

$$
D(V, R) \subseteq Y \subseteq\{z \in Z \mid z(A)=(0), z(V) \subseteq R\} .
$$


$R$ is just the radical of the bilinear form $\langle$,$\rangle . Also$

$$
Z_{0}=\{z \in Z \mid z(A)=0, z(V)=0\}
$$

and

$$
A \oplus V \oplus D(V, V)+\left[A^{*}, A^{*}\right] \oplus A^{*} \oplus \bar{V} \oplus \bar{A}
$$

are ideals of $L$.

LeMma 2.10. Let $L$ be $a 5$ and 7 torsion-free *-Lie algebra such that $R=(0)$ and $Z_{0}=(0)$. Then $L$ has absolute zero divisors if and only if $A$ does.

Proof. Every absolute zero divisor of $A$ is an absolute zero divisor of $L$. For the other direction let $\Omega$ be the set of absolute zero divisors of $L$ and assume $\Omega \neq(0)$. The projection of an element of $\Omega$ onto $A$ is an absolute zero divisor of $A$, and likewise for $\bar{A}$. So suppose $\Omega \subseteq V \oplus A^{*} \oplus Z \oplus \bar{V}$, and observe that Lie automorphisms of $L$ leave the set $\Omega$ invariant. The element $x=v+z+d^{*}+\bar{w} \in \Omega$ if and only if $\exp D_{e}(x)=v+z+d^{*}-d+\bar{w}$ $+w \in \Omega$. So by the above remarks we can assume $d=0$ and $\Omega \subseteq V \oplus Z \oplus$ $\bar{V}$. For each $u \in V$ the projection of $\exp D_{u}(x) \in \Omega$ on $A^{*}$ is $\langle u, w\rangle$ which must be zero. But the nondegeneracy of the form then implies $w=0$, and similarly $v=0$. The only remaining possibility is $\Omega \subseteq Z$. Applying $\exp D_{a}$ and $\exp D_{u}$ for $a \in A, u \in V$ to $z \in \Omega \subseteq Z$ shows $z \in Z_{0}$. So it must be $\Omega \neq 0$ forces $A$ to have nonzero absolute zero divisors.

LEMMA 2.11. Assume $L$ is a ${ }^{*}$-Lie algebra.

(1) If $Z=D(V, V)+\left[A^{*}, A^{*}\right]$ and $Z_{0}=R=(0)$, then $A$ a simple Jordan algebra implies $L$ is a simple Lie algebra.

(2) If $L$ is simple and Artinian then $A$ is a simple Jordan algebra, or $A=S \otimes k\left[x_{1}, \ldots, x_{n}\right]$ where $S$ is a simple Jordan algebra of prime characteristic and $k\left[x_{1}, \ldots, x_{n}\right]$ is a ring of truncated polynomials.

(3) If $L$ is simple, nondegenerate, and Artinian, then $A$ is simple.

Proof. According to Lemma $2.9 A$ is derivation simple if $L$ is simple, and $A$ possesses a minimal ideal if $L$ is Artinian. So, in (2) $A$ is asserted by a result of Block [4]. Now $A$ has absolute zero divisors in (2) unless $A$ is simple, so (3) follows, and (1) is just a direct consequence of Lemma 2.9.

TheOREM 2.12. Let $L$ be a nondegenerate, Artinian *-Lie algebra. Suppose $L=A \oplus V \oplus A^{*} \oplus Z \oplus \bar{V} \oplus \bar{A}$ where $Z=D(V, V)+\left[A^{*}, A^{*}\right]$ and $\langle$, is nondegenerate. Then $L$ is the direct sum of a finite number of simple Lie algebras which satisfy these hypotheses; and the converse holds also.

Proof. We know from Lemma 2.6(3) that we can write $A=I_{1} \oplus \cdots \oplus I_{t}$ where $I_{j}$ are simple ideals of $A$. It is easy to verify that the $I_{j}$ are left invariant by derivations of $A$; hence by $Z$. We show $L=\bigoplus \Sigma L_{j}$ where 


$$
L_{j}=I_{j} \oplus I_{j} V \oplus D\left(V, I_{j} V\right)+\left[A^{*}, I_{j}^{*}\right] \oplus I_{j}^{*} \oplus \overline{I_{j} V}+\bar{I}_{j} .
$$

Because each $I_{j}$ is a simple Jordan algebra satisfying the minimum condition, it has a multiplicative identity $e_{j}$. Now for any representation $\rho$ of $A$ one has from Jacobson [11, p. 96] that

$$
\left[a^{\rho},(b \cdot c)^{\rho}\right]+\left[b^{\rho},(c \cdot a)^{\rho}\right]+\left[c^{\rho},(a \cdot b)^{\rho}\right]=0 .
$$

This identity with the specialization $a=a_{i} \in I_{i}, b=a_{j} \in I_{j}$, and $c=e_{j}$ shows $I_{j}\left(I_{i} V\right)=(0)$ for $i \neq j$. This in turn leads to $I_{j} V \cap I_{i} V=(0)$ for $i \neq j$, and, consequently, $L=\bigoplus \Sigma L_{j}$. To argue each $L_{j}$ is simple we need only invoke Lemma 2.10. The rest is straightforward to verify.

Theorem 2.12 says that in many instances the study of nondegenerate, Artinian *-Lie algebras can be reduced to considering simple algebras. One place to begin is with finite dimensional simple Lie algebras over algebraically closed fields. The characteristic 0 case has been studied extensively, so the next section investigates nondegenerate finite dimensional simple *-Lie algebras over algebraically closed fields of prime characteristic.

3. Ad-nilpotent elements and classical Lie algebras. It has been the goal of work of Kostrykin and Jacobs to determine whether the existence of nonzero absolute zero divisors is a necessary and sufficient condition for distinguishing the nonclassical from the classical simple Lie algebras over algebraically closed fields of prime characteristic. To this end Kostrykin [15] proved

THEOREM 3.1. In a finite dimensional simple Lie algebra over an algebraically closed field $F$ of characteristic $p>5$, assume the following conditions are satisfied:

(i) There is a Cartan decomposition $L=H \oplus \Sigma_{\alpha \neq 0} L_{\alpha}$ relative to a Cartan subalgebra $H$ of $L$ such that $D_{a}^{p-1}=0$ for some $a \neq 0$ in $H$ or $L_{\alpha}$.

(ii) $L$ is nondegenerate.

(iii) $L$ is a p-Lie algebra.

Then $L$ is classical.

Later in [7] Jacobs was able to remove hypothesis (iii). We will replace (i) with the assumption that $L$ contain an $x \neq 0$ with $D_{x}^{p-1}=0$ and omit (iii) as well. This is advantageous for condition (i) is not an invariant of the Lie algebra, and hence, necessitates making a judicious choice of the Cartan subalgebra. This result was originally proved using Jacobs' theorem by the author in her doctoral dissertation and, independently, by Strade [18]. However, the investigations of *-Lie algebras in the preceding section give us a means of attacking the problem directly, thereby eliminating or simplifying many of calculations in Kostrykin's and Jacobs' papers. In addition to results on *-Lie algebras our approach will use a theorem due to Block [3] to prove a sequence of steps which lead to 
THEOREM 3.2. Let $L$ be a finite dimensional simple Lie algebra over an algebraically closed field $F$ of characteristic $p>5$. Assume:

(i) there is an $x \neq 0$ in $L$ such that $D_{x}^{p-1}=0$;

(ii) $L$ is nondegenerate.

Then $L$ is classical.

Step 1. Under the hypotheses of the theorem $L$ is a *-Lie algebra and there is a decomposition $L=V_{2} \oplus V_{1} \oplus V_{0} \oplus V_{-1} \oplus V_{-2}$ such that $V_{2}, V_{-2}$ are 1-dimensional.

Proof. By Proposition 1.5 there is a $c \neq 0$ such that $D_{c}^{3}=0$ and $D_{c}^{2}(L)$ is an inner ideal. Using the same argument as in the proof of Lemma 2.8, we see $L$ is a *-Lie algebra, and, moreover, there are elements $e, f, h$ in $L$ with $e \in D_{e}^{2}(L)=V_{2}$ and $D_{e}^{3}=0$ such that the decomposition of $L$ into eigenspaces relative to $D_{h}$ is given by $L=V_{2} \oplus V_{1} \oplus V_{0} \oplus V_{-1} \oplus V_{-2}$ where $V_{2}$ is a Jordan division algebra. But then $V_{2}=F e$ and $V_{-2}=F \bar{e}$ for $\bar{e}=f$ since $F$ is algebraically closed.

Now let $H$ be a Cartan subalgebra of $V_{0}$. The element $h$ centralizes $V_{0}$, so it must belong to $H$. Therefore $H$ is in fact a Cartan subalgebra of $L$ which leaves each $V_{i}$ invariant, and accordingly each $V_{i}$ decomposes into root spaces relative to $H$. The space $V_{2}$ corresponds to a root which we will denote by $\alpha$, and $V_{-2}$ belongs to the root $-\alpha$. Our next objective will be to show that the root spaces of $V_{1}$ and $V_{-1}$ are also 1-dimensional. The steps will follow the general outline used by Kostrykin [15].

For $u, v \in V_{1}$ we will write $[u, v]=\langle u, v\rangle e$ so that if $\bar{u}, \bar{v} \in V_{-1}$, then $[\bar{u}$, v] $=-\langle u, v\rangle \bar{e}$. The symbol $V_{i, \delta}$ will denote the root space of $V_{i}$ corresponding to the root $\delta$. On numerous occasions we will be concerned with an element $s$ with the property that $D_{s}^{2}(L)=F s$. In this situation there is a $t$ such that $D_{s}^{2}(t)=-2 s$ and $D_{[s, t]}$ is a semisimple transformation with eigenvalues among $\pm 2, \pm 1,0$. The decomposition of $L$ relative to $[s, t]$ will be given by

$$
L=V_{2}(s) \oplus V_{1}(s) \oplus V_{0}(s) \oplus V_{-1}(s) \oplus V_{-2}(s)
$$

where $V_{2}(s)=F_{s}, V_{-2}(s)=F t$, and $V_{0}(s)=Z(s) \oplus F[s, t]$. We will make use of the following result of Kostrykin [15]:

Proposition 3.3. If $u \in V_{1}$ and $D_{u}^{i}(\bar{e})=0$ for $i=3$ or 4 , then $D_{u}^{i}=0$.

Step 2. If $0 \neq u \in V_{1, \gamma}$ and $D_{u}^{2}(\bar{e})=0$, then $V_{1, \gamma}$ is 1-dimensional.

Proof. In order to obtain a decomposition as above we first show $D_{u}^{2}(L)=$ $F u$. We need only consider $D_{u}^{2}$ on $V_{0}=Z \oplus F h$ and on $V_{-1}$ because it is zero everywhere else. Now $D_{u}^{2}(h)=-D_{u}(u)=0$, and for every $z \in Z$, 


$$
0=D_{u}^{3}([z, \bar{e}])=3\left[D_{u}^{2}(z), 3\left[D_{u}^{2}(z), D_{u}(\bar{e})\right]\right]=-3\langle z(u), u\rangle u .
$$

Therefore

$$
\langle z(u), u\rangle=0 \text { and } D_{u}^{2}(z)=\langle z(u), u\rangle e=0
$$

for every $z \in Z$. For $\bar{v} \in V_{-1}$,

$$
D_{u}^{2}(\bar{v})=D_{u}^{2}([\bar{e}, v])=2\left[D_{u}(\bar{e}), D_{u}(v)\right]=2\langle u, v\rangle u .
$$

Consequently, $D_{u}^{2}(L) \subseteq F u$, and since $L$ is nondegenerate, $D_{u}^{2}(L)=F u$ necessarily holds. We can take $\bar{w} \in V_{-1,-\gamma}$ so that $D_{u}^{2}(\bar{w})=-2 u$ and $D_{[u, \bar{w}]}$ is semisimple with eigenvalues among $\pm 2, \pm 1,0$. The element $[u, w]$ belongs to $H$ and $\gamma([u, w])=2$. From this it follows that $V_{1, \gamma} \subseteq V_{2}(u)=F u$.

Step 3. Assume there is some $0 \neq u \in V_{1, \gamma}$ with $D_{u}^{3}=0$. Then $V_{1, \gamma}$ is 1-dimensional.

Proof. We suppose $D_{u}^{2}(\bar{e})=b \neq 0$ and proceed to show $D_{b}^{2}(L)=F b$. The element $b$ belongs to $V_{0}$, and according to Lemma $1.7, D_{b}^{3}=0, D_{b}^{2}=$ $D_{u}^{2} D_{\bar{e}}^{2} D_{u}^{2}$. Therefore

$$
D_{b}^{2}(L) \subseteq D_{u}^{2}(L) \subseteq F e \oplus V_{1} \oplus V_{0}
$$

so that $b$ must belong to $Z$. Now $\overline{b^{2}(e)}=b^{2}(\bar{e})=0$ and $\overline{b^{2}(v)}=b^{2}(\bar{v})=0$. This shows that $D_{b}^{2}(L)=D_{b}^{2}(Z)$. For $z \in Z$,

$$
\begin{aligned}
D_{b}^{2}(z) & =D_{u}^{2} D_{\bar{e}}^{2} D_{u}^{2}(z)=D_{u}^{2} D_{\bar{e}}^{2}(\langle z(u), u\rangle e) \\
& =-2\langle z(u), u\rangle D_{u}^{2}(\bar{e})=-2\langle z(u), u\rangle b .
\end{aligned}
$$

Thus $D_{b}^{2}(L)=F b$. The element $b$ belongs to $V_{0, \gamma-2 \alpha}$ so there exists an $a \in Z$ with $a \in V_{0,-\gamma+2 \alpha}$ such that $D_{b}^{2}(a)=-2 b$ and $D_{[b, a]}$ is semisimple with eigenvalues among $\pm 2, \pm 1,0$. Since $[b, a] \in H$ and $\gamma-2 \alpha([b, a])=2$, $V_{0, \gamma-2 \alpha} \subseteq V_{2}(b)=F b$. For every $v, w \in V_{1, \gamma}, D_{v} D_{w}(\bar{e}) \in V_{0,2 \gamma-\alpha}$, so $D_{v} D_{w}(\bar{e})$ is a scalar multiple of $b$. If $V_{1, \gamma}$ is more than 1-dimensional there would be an $x \neq 0$ in $V_{1, \gamma}$ with $D_{x}^{2}(e)=0$. Step 1 would give the contradiction that $V_{1, \gamma}$ is 1-dimensional. So we are forced to conclude $V_{1, \gamma}$ is 1-dimensional.

Step 4. $V_{1, \gamma}$ is 1-dimensional for each root $\gamma$ of $V_{1}$.

ProOF. In view of the previous steps and Kostrykin's result we can suppose $D_{t}^{3}(\bar{e}) \neq 0$ for every $t \in V_{1, \gamma}$. Let us assume initially that there is some $v \in V_{1, \gamma}$ with $D_{v}^{4}=0$. Now $u=D_{v}^{3}(e)$ is ad-nilpotent of index 3 so the root space $V_{1,3 \gamma-\alpha}$ to which it belongs is 1-dimensional. If $V_{1, \gamma}$ is more than 1-dimensional there is an $x \neq 0$ in $V_{1, \gamma}$ with $D_{x}^{3}(\bar{e})=0$. Thus we would contradict the assumption that $D_{t}^{3}(\bar{e}) \neq 0$ for any $t \in V_{1}$, unless $V_{1, \gamma}$ is 1-dimensional. It is sufficient to suppose then that for every $v \in V_{1, \gamma} D_{v}^{4} \neq 0$. By Kostrykin's result $0 \neq D_{v}^{4}(\bar{e}) \in V_{2}=F e$. If $V_{1, \gamma}$ has two independent 
elements we can find an $x \neq 0$ in $V_{1, \gamma}$ with $D_{x}^{4}(e)=0$. Consequently, in this case also, $V_{1, \gamma}$ is 1-dimensional.

Step 5 (COROLlaRY). Each root space of $V_{-1}$ is 1-dimensional.

Step 6 (COROLLARY). For every root $\gamma$ of $V_{1}$ and every $u \in V_{1, \gamma}, D_{u}^{4}=0$.

Proof. By Kostrykin's result we can assume $0 \neq D_{u}^{4}(e)=\xi$ e. Then $2 \gamma=\alpha$ and $D_{u}^{3}(\bar{e}) \in V_{1, \gamma}$. Hence $D_{u}^{3}(\bar{e})=\lambda u$ by the 1-dimensionality of $V_{1, \gamma}$. But then $D_{u}^{4}(\bar{e})=D_{u}(\lambda u)=0$, contrary to assumption.

Next we specialize to the case $V_{0}=\left[V_{2}, V_{-2}\right]+\left[V_{1}, V_{-1}\right]$. This is always true if $L$ is simple for $V_{2} \oplus V_{1} \oplus\left[V_{2}, V_{-2}\right]+\left[V_{1}, V_{-1}\right] \oplus V_{-1} \oplus V_{-2}$ is an ideal of $L$. We have not as yet used the hypothesis of simplicity, and indeed all we will use to prove Theorem 3.2 will be the weaker assumption that $V_{0}$ has this form and the next theorem due to Block.

THEOREM 3.4 (BLock [3]). Assume $L$ is a finite dimensional Lie algebra over an algebraically closed field $F$ of characteristic $p>5$, and suppose $H$ is a Cartan subalgebra of $L$ such that:

(i) $L^{2}=L$.

(ii) The center of $L$ is (0).

(iii) For every nonzero root $\beta$ of $L$ relative to $H, L_{\beta}$ is 1-dimensional and $\beta\left(\left[L_{\beta}, L_{-\beta}\right]\right) \neq 0$.

Then $L$ is the direct sum of simple classical algebras or Albert-Zassenhaus algebras.

Under assumptions (i) and (ii) of Theorem 3.2 and the hypothesis $V_{0}=$ $\left[V_{2}, V_{-2}\right]+\left[V_{1}, V_{-1}\right]$, it is clear that (i) and (ii) of Block's theorem are satisfied. Moreover, we have shown thus far that the root spaces of $V_{2}, V_{-2}$, $V_{1}, V_{-1}$ are 1-dimensional and, for the root $\alpha$, (iii) holds.

Step 7. For every root $\gamma$ of $V_{1},-\gamma$ is a root of $V_{-1}$ and

$$
\gamma\left(\left[V_{1, \gamma}, V_{-1,-\gamma}\right]\right) \neq 0 \text {. }
$$

Proof. If $0 \neq u \in V_{1, \gamma}$ and $D_{u}^{2}(\bar{e})=0$, then as was observed in the proof of Step 2, there is a $\bar{w} \in V_{-1,-\gamma}$ so that $[u, \bar{w}] \in H$ and $D_{[u w]}$ is semisimple with eigenvalues $\pm 2, \pm 1,0$. Also $\langle u, w\rangle=-1$ and $\gamma([u, \bar{w}])=2$ in this case.

If $0 \neq u \in V_{1, \gamma}$ and $D_{u}^{3}=0$, but $D_{u}^{2}(e)=b \neq 0$, then from Step 3 there is an $a \in Z$ with $D_{b}^{2}(a)=-2 b$. In addition, $[b, a] \in H$ and $D_{[b, a]}$ is semisimple with eigenvalues $\pm 2, \pm 1,0$. Now since $u$ is a root vector $[[b, a] u]$ is a multiple of $u$. The fact that $u$ commutes with $b$ implies that $u \in V_{1}(b)$ or $u \in Z(b)$. We note from Step 3 that $D_{b}^{2}(a)=-2\langle a(u), u\rangle b=-2 b$, so $a(u) \neq 0$ and $\langle a(u), u\rangle=1$. Thus $u \notin Z(b)$ and $u \in V_{1}(b)$. The element $0 \neq \overline{a(u)}$ belongs to $V_{-1,-\gamma}$ and we proceed to show $\gamma([u, \overline{a(u)}]) \neq 0$. A computation of $[b, a]$ yields: 


$$
[b, a]=[a(u), \bar{u}]+[u, a(\bar{u})]=2 D(u, a(u)) \text {. }
$$

From §2,

$$
[u, \overline{a(u)}]=D(u, a(u))-\langle u, a(u)\rangle^{*}=\frac{1}{2}[b, a]+\frac{1}{2} h .
$$

Thus

showing $\gamma([u, \overline{a(u)}]) \neq 0$.

$$
[[u, \overline{a(u)}] u]=\left[\frac{1}{2}[b, a]+\frac{1}{2} h, u\right]=u,
$$

In light of these reductions we can suppose $u \in V_{1, \gamma}$ and $D_{u}^{3}(e)=s \neq 0$ where $s$ belongs to $V_{1,3 \gamma-\alpha}$. From Step 6 and Kostrykin's result mentioned in $\S 1$ it follows that $D_{s}^{3}=0$ so that $s$. is as in the first or second paragraph. When $D_{D_{u}^{4}(e)}=0$ is applied to $\bar{e}$, one obtains $D_{u} D_{\bar{e}} D_{u}^{3}(\bar{e})=0$. Expanding $D_{s}$ in terms of $D_{u}, D_{\bar{e}}$ and using this result, one can show that $D_{s}^{2}(\bar{e})=0$. Therefore, according to the first paragraph, there is a $\bar{t} \in V_{-1,-3 \gamma+\alpha}$ with $[s$, $\bar{t}] \in H, D_{[s, \bar{l}]}$ semisimple with eigenvalues $\pm 2, \pm 1,0,\langle s, t\rangle=-1$, and $3 \gamma-\alpha([s, \bar{t}])=2$. We will prove that $0 \neq D_{u}^{2}(\bar{t}) \in V_{-1,-\gamma}$ and that

$$
\gamma\left(\left[u, \overline{D_{u}^{2}(\bar{t})}\right]\right) \neq 0 .
$$

To begin, observe that $[[s, \bar{t}] e]=-\langle s, t\rangle e=e$ so that $e \in V_{1}(s)$ and $\gamma([s, \bar{t}])=1$ and, as a further consequence, $\gamma([s, \bar{t}])=1$. For each root $\beta \neq 3 \gamma-\alpha, V_{1, \beta}$ is orthogonal to $V_{1,-3 \gamma+2 \alpha}$ relative to $\langle$,$\rangle . Therefore [ u$, $t]=\langle u, t\rangle e=0$ provided $\gamma \neq 3 \gamma-\alpha$. However, it is true that $\gamma \neq 3 \gamma-\alpha$ in this case since $\gamma([s, \bar{t}])=1=\alpha([s, \bar{t}])$. Because $[u, t]=0$ the mappings $D_{u}$ and $D_{t}$ commute. This gives

$$
0 \neq\langle s, t\rangle e=\left[D_{u}^{3}(\bar{e}), t\right]=D_{t} D_{u}^{3}(\bar{e})=-D_{u}^{3} D_{t}(\bar{e})=\left[u, D_{u}^{2}(\bar{t})\right] .
$$

Thus $\overline{D_{u}^{2}(\bar{t})} \neq 0$, and $\gamma\left(\left[u, \overline{\left.D_{u}^{2}(t)\right]}\right) \neq 0\right.$ will follow from this calculation:

$$
\begin{aligned}
{[s, \bar{t}] } & =\left[D_{u}^{3}(\bar{e}), \bar{t}\right]=\left(3 D_{u} D_{\bar{e}} D_{u}^{2}-D_{\bar{e}} D_{u}^{3}\right)(\bar{t}) \\
& =3\left[u, \overline{D_{u}^{2}(\bar{t})}\right]-D_{\bar{e}} D_{u}^{3}(\bar{t})=3\left[u, \overline{D_{u}^{2}(\bar{t})}\right]-D_{\bar{e}}\left(\left[D_{u}^{3}(\bar{e}), t\right]\right) \\
& =3\left[u, \overline{D_{u}^{2}(\bar{t})}\right]-h .
\end{aligned}
$$

Therefore $\left[u, \overline{D_{u}^{2}(\bar{t})}\right]=([s, \bar{t}]+h) / 3$ and $\gamma\left(\left[u, \overline{D_{u}^{2}(\bar{t})}\right]\right)=2 / 3$.

It remains to be shown that the nonzero root spaces of $V_{0}$ are 1-dimensional, and for every root space $V_{0, \delta}$ of $V_{0}, V_{0,-\delta}$ is also a root space with $\delta\left(\left[V_{0, \delta}, V_{0,-\delta}\right]\right) \neq 0$. Under the assumption that $V_{0}=\left[V_{2}, V_{-2}\right]+\left[V_{1}, V_{-1}\right]$, the elements $[u, v]$ for $u \in V_{1, \gamma}$ and $\bar{v} \in V_{-1,-\beta}$ with $\beta \neq \gamma$ generate these root spaces. It suffices to show that there exist root vectors $q, r$ with $D_{q}^{2}(L)=F_{q}$ and $[q, r] \in H$ such that $[u, v] \in V_{i}(q)$ for some $i= \pm 1$ or \pm 2 . For if $\delta=\gamma-\beta$, then $\delta([q, r])=i$ implies $V_{0, \delta} \subseteq V_{i}(q)$. All our previous 
considerations applied to the decomposition

$$
L=F q \oplus V_{1}(q) \oplus V_{0}(q) \oplus V_{-1}(q) \oplus F r
$$

instead of to the one determined by $e, f, h$ yield $V_{0, \delta}$ is 1-dimensional and $\delta\left(\left[V_{0, \delta}, V_{0,-\delta}\right]\right) \neq 0$.

Step 8. Each nonzero root of $V_{0}$ satisfies hypothesis (iii) of Block's theorem.

PRoof. Let $0 \neq[u, v] \in V_{0, \delta}$ where $u \in V_{1, \gamma}, \bar{v} \in V_{-1,-\beta}$ and $\gamma \neq \beta$. The first observation to make is that $[u, v]=0$. Indeed if $[u, v] \neq 0$, then $\gamma-\beta+\alpha=\alpha$ and $\gamma=\beta$ contrary to assumption. Thus the product $[u, v]$ is symmetric in $u$ and $v$ because $[u, v]=D(u, v)=[v, u]$.

Let us restrict first to the case $D_{u}^{2}(\bar{e})=0$. Then there is $\bar{w} \in V_{-1,-\gamma}$ such that

$$
L=F u \oplus V_{1}(u) \oplus V_{0}(u) \oplus V_{-1}(u) \oplus F \bar{w} .
$$

Relative to this decomposition $\bar{v} \in V_{1}(u), V_{0}(u)$ or $V_{-1}(u)$. Now $\bar{v} \in V_{1}(u)$ can be ruled out since $[u, v] \neq 0$. If $\bar{v} \in V_{0}(u)$ then $[u, v] \in V_{2}(u)=F u \subseteq$ $V_{1}$ which contradicts $[u, v] \in V_{0}$. So $\bar{v} \in V_{-1}(u)$ necessarily holds and $[u$, v] $\in V_{1}(u)$ in this instance.

Because of the symmetry of $[u, \bar{v}]$ we can assume $D_{u}^{2}(\bar{e}) \neq 0, D_{v}^{2}(\bar{e}) \neq 0$. Let us suppose $D_{u}^{3}=0$ so that $b=D_{u}^{2}(e) \in Z$ leads to the decomposition

$$
L=F b \oplus V_{1}(b) \oplus V_{0}(b) \oplus V_{-1}(b) \oplus F a .
$$

Recall that $u \in V_{1}(b)$ and $e, \bar{e}, h \in Z(b)$ in this situation so that $\bar{v}$ must be in $V_{1}(b), V_{0}(b)$ or $V_{-1}(b)$. In case $\bar{v} \in V_{0}(b)$, then $[u, v] \in V_{1}(b)$ as desired. On the other hand if $\bar{v} \in V_{1}(b)$, then $[u, \bar{v}] \in V_{2}(b)$. Lastly in the event $\bar{v} \in$ $V_{-1}(b), v \in V_{-1}(b)$ also, and $0 \neq D_{v}^{2}(\bar{e}) \in V_{-2}(b)$. But this says $-2 \beta+\alpha$ $=-2 \gamma+\alpha$, and $\beta=\gamma$ contrary to assumption.

In the final consideration $D_{u}^{3} \neq 0$ and $D_{v}^{3} \neq 0$. The element $s=D_{u}^{3}(\bar{e})$ gives the decomposition

$$
L=F s \oplus V_{1}(s) \oplus V_{0}(s) \oplus V_{-1}(s) \oplus F \bar{t}
$$

where both $u, e$ are in $V_{1}(s)$ and $\bar{e} \in V_{-1}(s)$. Now $v$ belongs to some $V_{i}(s)$ but not to $V_{2}(s)=F s$ since $D_{s}^{3}=0$. Also $v \notin F t$ since they are different eigenspaces relative to $h$. If $v \in V_{1}(s)$, then $\bar{v} \in V_{0}(s)$ and [uv] $\in V_{1}(s)$. If $v \in V_{-1}(s)$, then $\bar{v} \in V_{-2}(s)$ and $[u, v] \in V_{-1}(s)$. The final case is $v \in$ $V_{0}(s)$. But then $D_{v}^{3}(\bar{e}) \in V_{-1}(s)$ and so $0 \neq\left[s, D_{v}^{3}(\bar{e})\right]=\left\langle s, D_{v}^{3}(\bar{e})\right\rangle$. This leads to $3 \gamma-\alpha-3 \beta+2 \alpha=\alpha$. That is, $\beta=\gamma$, contrary to assumption. Thus, in all possible situations the nonzero root spaces satisfy Block's assumptions.

To conclude the proof of Theorem 3.2, we need only eliminate the possibility of having Albert-Zassenhaus algebras. However, by the proof of Step 7, $H$ has a basis of elements whose adjoint mappings are semisimple with 
eigenvalues $\pm 2, \pm 1,0$. The proof of Block's theorem shows that if the algebra is not classical then there is a root $\alpha \neq 0$ such that $\alpha, 2 \alpha, \ldots$, $(p-1) \alpha$ are all roots. But then, since $p>5, \alpha(g)=0$ for every $g \in H$, contradicting the fact that $H$ is self-normalizing. So it must be that algebras satisfying the hypotheses of Theorem 3.2 are classical, and each classical algebra satisfies these hypotheses.

Kostrykin [14] has been concerned with conditions under which the degeneracy of the Killing form implies the algebra has nonzero absolute zero divisors. In light of Theorem 3.2 we have the following:

THEOREM 3.5. Let $L$ be a finite dimensional simple Lie algebra over an algebraically closed field of characteristic $p>5$. Assume:

(i) there is an $x \neq 0$ such that $D_{x}^{p-1}=0$; and

(ii) the Killing form of $L$ is degenerate.

Then $L$ has nonzero absolute zero divisors unless $L$ is classical of type $A_{n}$ or $C_{n}$ for $p$ dividing $n+1$, of type $B_{n}$ for $p$ dividing $2 n-1$ or of type $D_{n}$ for $p$ dividing $n-1$.

\section{BIBLIOGRAPHY}

1. B. N. Allison, $A$ construction of Lie algebras from J-ternary algebras, Amer. J. Math. 98 (1976), 285-294.

2. G. M. Benkart, The Lie inner ideal structure of associative rings, J. Algebra 43 (1976), 561-584.

3. R. E. Block, On the Mills-Seligman axioms for Lie algebras of classical type, Trans. Amer. Math. Soc. 121 (1966), 378-392. MR 32 \#5795.

4. Determination of the differentially simple rings with a minimal ideal, Ann. of Math. (2) 90 (1969), 433-459. MR 40 \# 4319.

5. W. Hein, A construction of Lie algebras by triple systems, Trans. Amer. Math. Soc. 205 (1975), 79-95.

6. U. Hirzebruch, Über eine Klasse von Lie-Algebren, J. Algebra 11 (1969), 461-467. MR 38 \#5869.

7. J. B. Jacobs, On classifying simple Lie algebras of prime characteristic by nilpotent elements, J. Algebra 19 (1971), 31-50. MR 46 \#223.

8. N. Jacobson, Enveloping algebras of semi-simple Lie algebras, Canad. J. Math. 2 (1950), 257-266. MR 13, 102.

9. __ A note on three dimensional simple Lie algebras, J. Math. Mech. 7 (1958), 823-831. MR 20 \#3901.

10. _ Lie algebras, Interscience, New York, 1962. MR 26 \# 1345.

11. __ Structure and representations of Jordan algebras, Amer. Math. Soc. Colloq. Publ., vol. 39, Amer. Math. Soc., Providence, R. I., 1968. MR 40 \# 4330.

12. A. I. Kostrikin, The Burnside problem, Izv. Akad. Nauk SSSR Ser. Mat. 23 (1959), 3-34; English transl., Amer. Math. Soc. Transl. (2) 36 (1964), 63-99. MR 24 \#A1947.

13. __ Simple Lie p-algebras, Trudy Mat. Inst. Steklov. 64 (1961), 79-89; English transl., Amer. Math. Soc. Transl. (2) 55 (1966), 195-206. MR 24 \# A1933.

14. (1963), 248-250 = Soviet Math. Dokl. 4 (1963), 637-640. MR 26 \#6224.

15. —_ Squares of adjoined endomorphisms in simple Lie p-algebras, Izv. Akad. Nauk SSSR Ser. Mat. 31 (1967), 445-487 = Math. USSR Izv. 1 (1967), 435-473. MR 36 \# 1501. 
16. K. McCrimmon, The radical of a Jordan algebra, Proc. Nat. Acad. Sci. U.S.A. 62 (1969), 671-678. MR 42 \#3137.

17. G. B. Seligman, Modular Lie algebras, Ergebnisse Math. Grenzgebiete, Bd. 40, SpringerVerlag, Berlin and New York, 1967. MR 39 \#6933.

18. H. Strade, Nonclassical simple Lie algebras and strong degeneration, Arch. Math. (Basel) 24 (1973), 482-485. MR 51 \# 12963.

19. J. Tits, Une classe d'algèbres de Lie en relation avec les algèbres de Jordan, Nederl. Akad. Wetensch. Proc. Ser. A 65 = Indag. Math. 24 (1962), 530-535. MR 26 \#3753.

Department of Mathematics, University of Wisconsin, Madison, Wisconsin 53706 (Current address)

Department of Mathematics, Yale University, New Haven, Connecticut 06520 